\title{
Melatonin induces anti-inflammatory effects via endoplasmic reticulum stress in RAW264.7 macrophages
}

\author{
YINA CHEN $^{1,2}$, QIAN ZHAO ${ }^{2}$, YANGJIE SUN ${ }^{2}$, YIN JIN $^{2}$, JIE ZHANG $^{3 *}$ and JIANSHENG WU ${ }^{2 *}$ \\ ${ }^{1}$ Department of Gastroenterology, Yuyao People's Hospital of Zhejiang, Yuyao, Zhejiang 315400, ${ }^{2}$ Department of \\ Gastroenterology, The First Affiliated Hospital of Wenzhou Medical University, Wenzhou, Zhejiang 325000; \\ ${ }^{3}$ Department of Neurology, Yuyao People's Hospital of Zhejiang, Yuyao, Zhejiang 315400, P.R. China
}

Received August 25, 2016; Accepted May 23, 2017

DOI: $10.3892 / \mathrm{mmr} .2018 .8613$

\begin{abstract}
Melatonin, which is predominantly secreted by the pineal gland and is released into the blood, appears to have anti-inflammatory properties. Several studies have shown that melatonin can relieve lipopolysaccharide-induced inflammatory responses of RAW264.7 cells. However, the mechanisms underlying this anti-inflammatory effect remain to be fully elucidated, particularly the association between melatonin and endoplasmic reticulum (ER) stress (ERS). Therefore, the present study examined the anti-inflammatory activity of melatonin in RAW264.7 cells and analyzed its molecular mechanisms in ERS. The RAW264.7 cells were stimulated by lipopolysaccharide and treated with melatonin. A Cell Counting Kit- 8 assay was used to assess the toxicity of melatonin. The degree of inflammation was evaluated using ELISA. The expression levels of ERS-associated protein molecules were examined using reverse transcription-quantitative polymerase chain reaction and western blot analyses. The results revealed that melatonin had no toxic effect on the RAW264.7 cells at the range of concentrations used in the experiment. Lipopolysaccharide stimulated the cells to produce inflammatory molecules; in the early stage, proteins associated with ERS increased, and then apoptosis occurred. The cells treated with melatonin exhibited attenuated inflammation, decreased expression of ERS-associated proteins and inhibition of apoptosis. Taken together, the results of the present study showed
\end{abstract}

Correspondence to: Mr. Jie Zhang, Department of Neurology, Yuyao People's Hospital of Zhejiang, 800 Chengdong Road, Yuyao, Zhejiang 315400, P.R. China

E-mail: jjtv138@126.com

Professor Jiansheng Wu, Department of Gastroenterology, The First Affiliated Hospital of Wenzhou Medical University, Building 1 Nanbaixiang Street, Ouhai, Wenzhou, Zhejiang 325000, P.R. China E-mail:wzwujs@163.com

*Contributed equally

Key words: melatonin, endoplasmic reticulum stress, inflammatory, RAW264.7 that melatonin may attenuate the inflammatory response by inhibiting the activation of ERS in RAW264.7 macrophages.

\section{Introduction}

The endoplasmic reticulum (ER) is one of the most important organelles in a cell; ER regulates calcium storage, lipid synthesis and protein folding. A physiological imbalance between the cellular demand for protein folding and the capacity of the ER to promote protein maturation leads to the accumulation of unfolded proteins in the ER lumen $(1,2)$. This can cause ER stress (ERS), which includes three aspects: i) Increase in the transcription and expression of ER proteins; ii) reduction in the speed of unfolded protein (UP) translation, preventing too many UPs from entering the ER; and iii) degradation of UPs by ER-associated degradation (3). This process is termed the unfolded protein response (UPR). The UPR is initiated by three ER transmembrane proteins: Basic leucine-zipper transcription factor activation of transcription factor 6 (ATF6), PKR-like ER-associated kinase (PERK) and endonuclease inositol-requiring enzyme 1 (IRE1) (4-6). When unfolded proteins aggregate in the ER lumen, they activate the ER membrane proteins and initiate the UPR. A moderate level of ERS is protective, however, when ERS becomes irreversible and normal functions cannot be restored, an apoptotic signal is initiated; in this way, the cells ultimately induce programmed cell death, or apoptosis. ERS-induced apoptosis includes the expression/activation of ERS-associated pro-apoptotic molecules, including C/EBP homologous protein (CHOP) and caspase 12 . These can activate apoptotic proteins downstream of ERS, including B-cell lymphoma 2 (Bcl-2)-associated X protein (Bax) and caspase 3 (7). Inflammation is one of the most common reactions in several diseases, and is a major factor leading to local environmental changes and the regulation of ERS in cells.

Melatonin $\left(\mathrm{C}_{13} \mathrm{H}_{16} \mathrm{~N}_{2} \mathrm{O}_{2}\right.$; MLT) is predominantly secreted by the pineal gland; however, the retina, tear ducts and skin can also produce low levels of melatonin in mammals (8-10). Melatonin is considered a compound with numerous potential applications due to its several biological functions, including improvement of sleep, combating aging, regulating immunity and suppressing tumors $(11,12)$. In addition, MLT can exhibit anti-oxidant and anti-inflammatory activities. Several studies have indicated that the nuclear factor $(\mathrm{NF})-\kappa \mathrm{B}$ pathway is 
important in inflammatory diseases. The administration of exogenous MLT causes anti-inflammatory effects via the $\mathrm{NF}-\kappa \mathrm{B}$ pathway (13), and studies have found that MLT can interfere with the $\mathrm{NF}-\kappa \mathrm{B}$ pathway in the lung tissues of an asthma rat model (14). This effect also occurs in stress ulcers (15) and inflammatory bowel disease (16).

However, whether MLT exerts its anti-inflammatory effect via ERS remains to be elucidated. The present study examined the potential role of MLT in controlling the ERS-associated signaling pathway to reduce inflammation in macrophages.

\section{Materials and methods}

Cell culture. The RAW264.7 macrophage cell line, purchased from the American Type Culture Collection (ATCC; Manassas, VA, USA), was cultured in DMEM/high glucose with $10 \%$ fetal bovine serum (FBS; Sigma-Aldrich; Merck KGaA, Darmstadt, Germany). The cells were incubated at $37^{\circ} \mathrm{C}$ under a humidified atmosphere of $5 \% \mathrm{CO}_{2}$. The nutrient solution was refreshed approximately every $48 \mathrm{~h}$, and the cells were passaged every 3-4 days.

Cell Counting Kit-8 (CCK-8) assay. Drug toxicity was assayed using a CCK-8 assay (Dojindo Molecular Technologies, Inc., Kumamoto, Japan) assay. A RAW264.7 cell suspension was seeded into 96 -well plates (100 $\mu \mathrm{l} /$ well; $1 \times 10^{4}$ cells/well). Following culture for $24 \mathrm{~h}$, the plating medium was replaced. The MLT was dissolved in fresh medium at different concentrations $(125,250$ and $500 \mu \mathrm{mol} / \mathrm{l})$. A control was supplemented in the culture medium. Each group consisted of three parallel wells. Following incubation for $24 \mathrm{~h}$, the CCK- 8 was added to the culture media, and a plate reader (Infinite ${ }^{\circledR} 200$ PRO NanoQuant; Tecan Austria GmbH, Grödig, Austria) was used to measure the supernatant in each well at a wavelength of $450 \mathrm{~nm}$. Each experiment was performed in triplicate. Cell viability in each group was calculated from the absorbance measured.

Cell treatment. A RAW264.7 cell suspension was seeded into 6 -well plates ( $2 \mathrm{ml} /$ well; $5 \times 10^{5}$ cells/well). Following culture for $24 \mathrm{~h}$, the plating medium was replaced with DMEM without 10\% FBS. After 24 h, MLT (Sigma-Aldrich; Merck KGaA) was dissolved in fresh medium at different concentrations $(125,250$ and $500 \mu \mathrm{mol} / \mathrm{l})$, whereas the cells in the control and lipopolysaccharide (LPS) group were incubated in culture medium. After $1 \mathrm{~h}$, LPS (Sigma-Aldrich; Merck KGaA) was added at a concentration of $1 \mu \mathrm{g} / \mathrm{ml}$ to the cells in the LPS and MLT $(125,250$ and $500 \mu \mathrm{mol} / \mathrm{l})$ groups for $6 \mathrm{~h}$. To investigate the effects of LPS on the ERS pathway for different durations of functional treatment, the cells were treated with $1 \mu \mathrm{g} / \mathrm{ml}$ LPS for $0,3,6,12$ and $24 \mathrm{~h}$.

ELISA. Following treatment, the culture supernatants of the cells were collected with the centrifugation $(2,500 \mathrm{x} g$ for $10 \mathrm{~min}$ at $4^{\circ} \mathrm{C}$ ). The concentrations of tumor necrosis factor (TNF)- $\alpha$, interleukin (IL)-1 and IL-6 in the culture supernatants were assessed using ELISA (R\&D Systems, Inc., Minneapolis, MN, USA) according to the manufacturer's instructions. The analyses were performed in triplicate in 96-well plates (Nunc; Gibco; Thermo Fisher Scientific, Inc., Waltham, MA, USA), which had been coated with $100 \mu \mathrm{l}$ aliquots of either anti-mouse TNF- $\alpha$ (cat. no. MAB4101), IL-1 (cat. no. MAB401) or IL-6 (cat. no. MAB406) (all from R\&D Systems, Inc.) monoclonal antibodies (dilutions for all 1:500) in phosphate-buffered saline (PBS) overnight at $4^{\circ} \mathrm{C}$. The plates were washed in PBS containing $0.05 \%$ Tween-20 and blocked with PBS containing $10 \%$ FBS for $2 \mathrm{~h}$. Following washing, the standards and the serum were added into the plates and incubated at room temperature for $3 \mathrm{~h}$. Following incubation, the wells were washed and $0.2 \mu \mathrm{g} / \mathrm{ml}$ the biotinylated anti-mouse TNF- $\alpha$, IL-1 or IL- 6 was added to each well. Incubation was continued at room temperature for $1 \mathrm{~h}$. The wells were washed, avidin-peroxidase was added, and the plates were incubated for $30 \mathrm{~min}$ at room temperature. The 3,5,3',5'-tetramethyl benzidine substrate was added following washing. To each well, sulfuric acid ( $2 \mathrm{~mol} / \mathrm{l})$ was added to terminate the reaction. The optical density of each well was read at a wavelength of $450 \mathrm{~nm}$.

Reverse transcription-quantitative polymerase chain reaction $(R T-q P C R)$ analysis. Following treatment with different concentrations of MLT and LPS for different durations, total RNA of the cells was isolated using TRIzol ${ }^{\mathrm{TM}}$ reagent, and synthesized to cDNA using a reverse transcription kit (both from Invitrogen; Thermo Fisher Scientific, Inc.). The primers were synthesized by Sangon Biotech Co., Ltd. (Shanghai, China) and the oligonucleotide sequences were as follows: Glucose-regulated protein 78 (GRP78) forward, 5'-AGCGAC AAGCAACCAAAGAT-3' and reverse, 5'-CCCAGGTCAAAC ACAAGGAT-3'; CHOP forward, 5'-ACAGAGGTCACACGC ACATC-3' and reverse, 5'-CTCCTGCTCCTTCTCCTTCA-3'; caspase 12 forward, 5'-CAATCTACAAGATCAAAGGTT TGGC-3' and reverse, 5'-CAAACTTTTTGTTGCAGATGA TGAG-3'; TNF- $\alpha$ forward, 5'-ACGGCATGGATCTCAAAG AC-3' and reverse, 5'-GTGGGTGAGGAGCACGTAGT-3'; $\beta$-actin forward, 5'-GTGCTATGTTGCTCTAGACTTCG-3' and reverse, 5'-ATGCCACAGGATTCCATACC-3'. The qPCR procedure $\left(10 \mathrm{~min}\right.$ at $95^{\circ} \mathrm{C}$ followed by 40 cycles of $95^{\circ} \mathrm{C}$ for $15 \mathrm{sec}$ and $60^{\circ} \mathrm{C}$ for $1 \mathrm{~min}$ ) was performed using a qPCR system (ABI 7500 system with SDS software 1.4; Applied Biosystems; Thermo Fisher Scientific, Inc.) and ABI Power SYBR-Green PCR Master mix (cat. no. 4367659). All reactions were repeated a minimum of three times. The mRNA expression levels were normalized against the housekeeping gene ( $\beta$-actin) using the ABI 7500 system with SDS 1.4 software and fold changes were calculated using the $2^{-\Delta \Delta \mathrm{Cq}}$ normalization method (17).

Western blot analysis. The cultured cells were homogenized in ice-cold RIPA buffer and phenylmethane sulfonyl fluoride (both from Beyotime Institute of Biotechnology, Shanghai, China) for $30 \mathrm{~min}$ on ice. The cells were scraped off the plate, and the extracts were transferred to a microcentrifuge tube and centrifuged at $1.2 \times 10^{4} \mathrm{x}$ g at $4^{\circ} \mathrm{C}$ for $20 \mathrm{~min}$. The protein concentration was determined using the BCA assay (Beyotime Institute of Biotechnology). Equal quantities of total protein (40 $\mu \mathrm{g})$ were subjected to $12 \%$ SDS-PAGE and then transferred onto polyvinylidene difluoride membranes (Bio-Rad Laboratories, Inc., Hercules, CA, USA) at $170 \mathrm{~mA}$ for $1-2 \mathrm{~h}$. The membranes were blocked at room temperature for $2 \mathrm{~h}$ in blocking buffer (TBS with $0.1 \%$ Tween-20 and 5\% non-fat milk) and then immunoblotted overnight at $4^{\circ} \mathrm{C}$ with primary antibodies targeted against the 
A

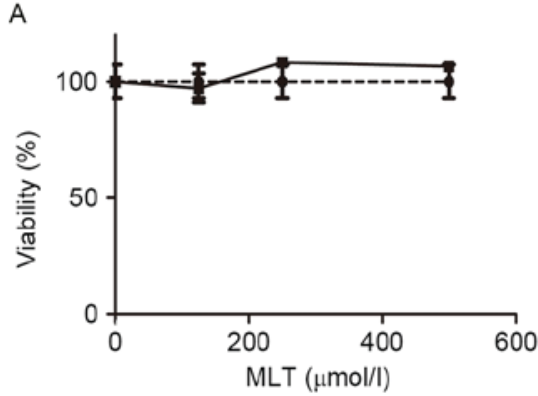

C

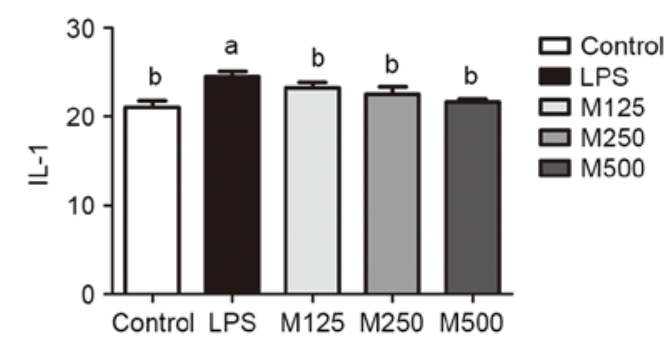

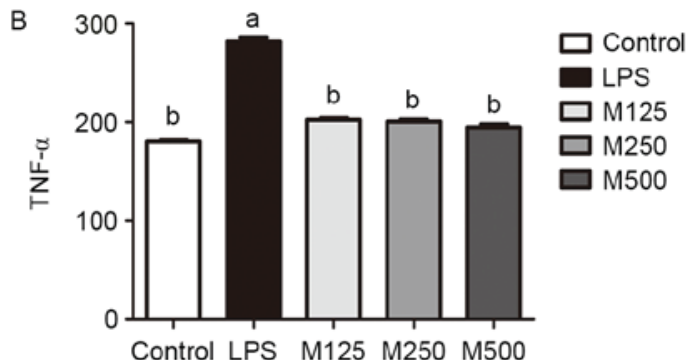

D

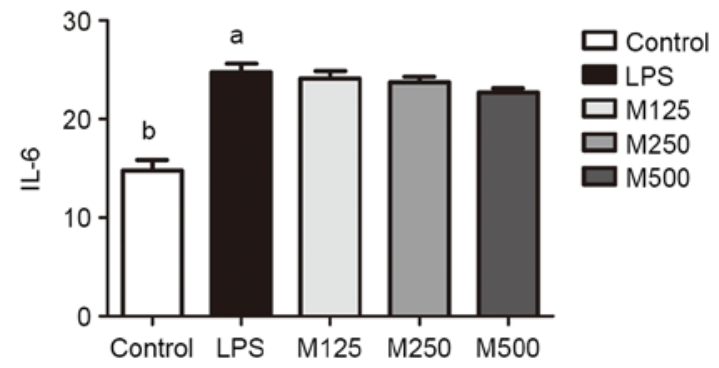

Figure 1. Toxicity of MLT towards RAW264.7 cells and its effects on attenuating the inflammatory reaction. (A) RAW264.7 cells were treated with various concentrations of MLT $(0,125,250$ and $500 \mu \mathrm{mol} / \mathrm{l})$. The Cell Counting Kit-8 assay results indicated that MLT at these concentrations was not toxic towards RAW264.7 cells with no statistically significant differences between groups ( $\mathrm{P}>0.05)$. Cells were pre-treated with MLT at concentrations of 125,250 and $500 \mu \mathrm{mol} / 1$ for $1 \mathrm{~h}$ in the M125, M250, and M500 groups, respectively. The LPS, M125, M250 and M500 groups were then incubated with $1 \mu \mathrm{g} / \mathrm{ml} \mathrm{LPS}$ for $6 \mathrm{~h}$. Culture supernatants were collected and analyzed for (B) TNF- $\alpha$, (C) IL-1 and (D) IL-6 using ELISA. ${ }^{\mathrm{a} P}<0.05$ vs. control group; ${ }^{\mathrm{b}} \mathrm{P}<0.05$ vs. LPS group. MLT, melatonin; LPS, lipopolysaccharide; TNF- $\alpha$, tumor necrosis factor- $\alpha$; IL, interleukin.

following: GRP78 (cat. no. ab108615), CHOP (cat. no. ab179823), caspase 12 (cat. no. ab10455), TNF- $\alpha$ (cat. no. ab6671) (all from Abcam, Cambridge, MA, USA), rabbit monoclonal Bcl-2 (D55G8; cat. no. 4223; Cell Signaling Technology, Inc., Beverly, MA, USA), Bax (Santa Cruz Biotechnology, Inc., Dallas, TX, USA), caspase 3 (cat. no. 9662; Cell Signaling Technology, Inc.), GAPDH (Santa Cruz Biotechnology, Inc.) and $\beta$-actin (Abcam) at dilutions of 1:1,000. Following washing three times with TBST for $10 \mathrm{~min}$, the membranes were incubated for $1 \mathrm{~h}$ at room temperature with goat anti-rabbit or goat anti-mouse secondary IgG conjugated to horseradish peroxidase $(1: 5,000$; Bioworld Technology, Inc., St. Louis Park, MN, USA) and then washed with TBST as previously. Finally, a Western Bright ECL detection kit (Advansta, Inc., Menlo Park, CA, USA) was used and the bands were detected. The density of specific bands was quantified using Image Lab software 4.1 (Bio-Rad Laboratories, Inc.) with an imaging densitometer (Bio-Rad ChemiDoc MP; Bio-Rad Laboratories, Inc.). The blots were subjected to densitometry using GAPDH or $\beta$-actin as internal controls.

Statistical analysis. All results were analyzed using SPSS software 13.0 (SPSS, Inc., Chicago, IL, USA). The data are reported as the mean \pm standard deviation. One-way analysis of variance was used for data comparisons. The Newman-Keuls post hoc test was used to compare the data in the presence of a significant difference. $\mathrm{P} \leq 0.05$ was considered to indicate a statistically significant difference.

\section{Results}

Toxicity of MLT towards RAW264.7 cells. The toxicity of MLT was assayed using a CCK-8 assay. The RAW264.7 cells were treated with MLT at different concentrations (125,

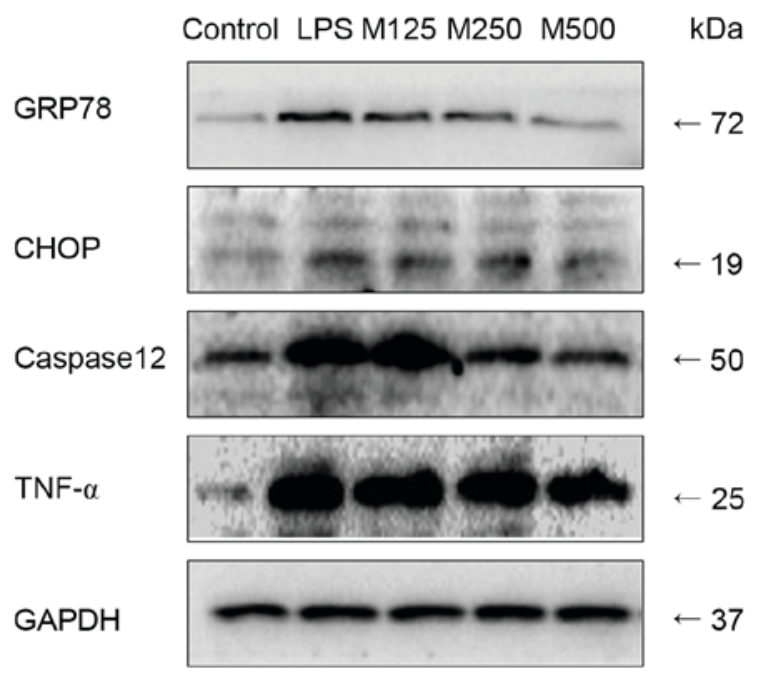

Figure 2. Effects of various concentrations of MLT on the endoplasmic reticulum stress pathway, determined using western blot analysis. Following treatment for $6 \mathrm{~h}$, cell lysates were subjected to SDS-PAGE followed by western blot analysis with anti-GRP78, anti-CHOP, anti-caspase12, anti-TNF- $\alpha$ and anti-GAPDH antibodies. The expression levels of all the above proteins were highest in the LPS group, and higher in the MLT groups, compared with the control group. The expression levels decreased as the concentration of MLT increased. LPS, lipopolysaccharide; MLT, melatonin; M125, $125 \mu \mathrm{mol} / 1 \mathrm{MLT}$; M250, $250 \mu \mathrm{mol} / 1 \mathrm{MLT}$; M500, $500 \mu \mathrm{mol} / 1 \mathrm{MLT}$; GRP78, glucose-regulated protein 78; $\mathrm{CHOP}, \mathrm{C} / \mathrm{EBP}$ homologous protein; TNF- $\alpha$, tumor necrosis factor- $\alpha$.

250 and $500 \mu \mathrm{mol} / \mathrm{l})$. These concentrations were selected according to previous experiments (18). The results indicated that, at these concentrations, MLT was not toxic towards the RAW264.7 cells (Fig. 1A). No significant differences were observed between the groups $(\mathrm{P}>0.05)$. 
A

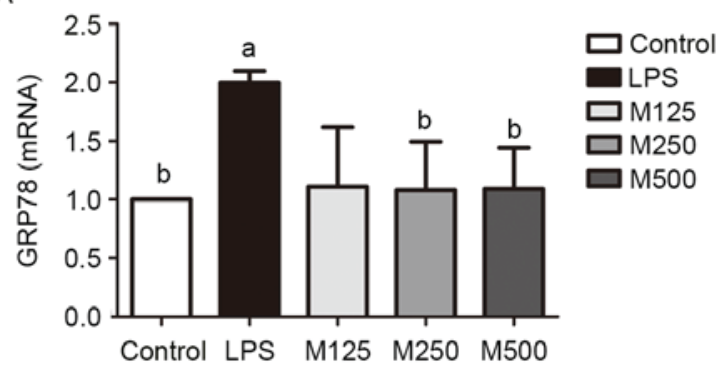

C

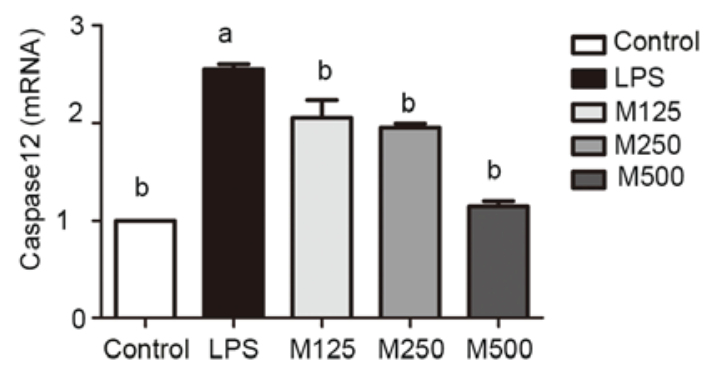

B

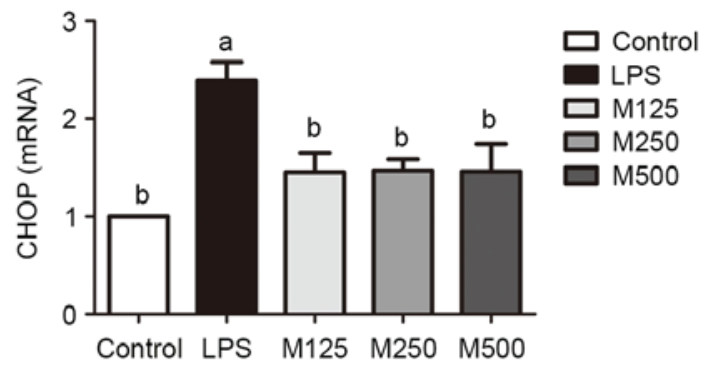

D

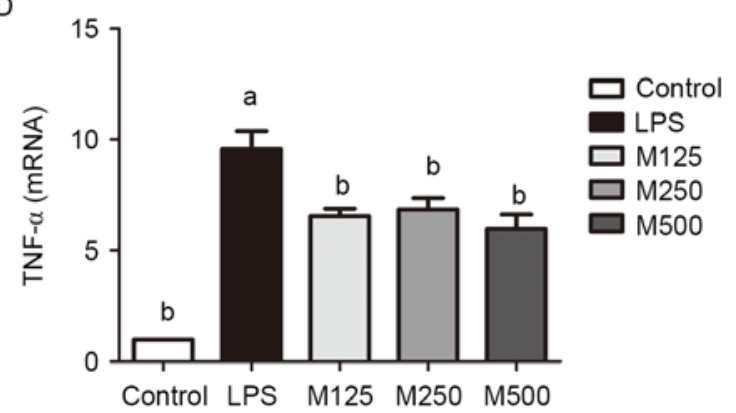

Figure 3. Effects of various concentrations of melatonin on the endoplasmic reticulum stress pathway, determined using RT-qPCR analysis. Total RNA from cells was isolated for RT-qPCR analysis of (A) GRP78, (B) CHOP, (C) caspase 12 and (D) TNF- $\alpha .{ }^{a} \mathrm{P}<0.05$ vs. control cells; ${ }^{b} \mathrm{P}<0.05$ vs. LPS. LPS, lipopolysaccharide; M125, $125 \mu \mathrm{mol} / 1$ melatonin; M250, $250 \mu \mathrm{mol} / 1$ melatonin; M500, $500 \mu \mathrm{mol} / 1$ melatonin; RT-qPCR, reverse transcription-quantitative polymerase chain reaction; GRP78, glucose-regulated protein 78; CHOP, C/EBP homologous protein; TNF- $\alpha$, tumor necrosis factor- $\alpha$.

Effects of MLT on attenuation of the inflammatory reaction. ELISA was used to evaluate the inflammatory indices of the differently treated groups. As shown in Fig. 1B-D, compared with the control, LPS induced inflammation $(\mathrm{P}<0.05)$. The inflammation in the MLT-treated groups was between that of the control and the LPS-treated groups $(\mathrm{P}<0.05)$. However, there were no statistically significant differences between the concentrations of MLT $(125,250$ and $500 \mu \mathrm{mol} / \mathrm{l})$.

Effects of concentrations of MLT on the ERS pathway. Western blot analysis and RT-qPCR analyses were used to determine the effects of MLT on ERS for the period of $6 \mathrm{~h}$. As shown in Figs. 2 and 3A-D, LPS provoked inflammation and activated ERS $(\mathrm{P}<0.05)$. Re-treatment with MLT relieved the inflammation and reduced the ERS $(\mathrm{P}<0.05)$. The extent of the inhibition was likely to be dependent on the concentration of MLT.

Effects of LPS on the ERS pathway at different durations of treatment. The expression levels of proteins associated with ERS were examined using RT-qPCR and western blot analyses of cells cultured with LPS for $0,3,6,12$ or $24 \mathrm{~h}$. The results showed that inflammation became more pronounced as duration was extended. The expression of TNF- $\alpha$ increased slowly as LPS was at a relatively low concentration, however, the increased mRNA expression of TNF- $\alpha$ was apparent at $6 \mathrm{~h}$. The expression levels of the ERS-associated molecules were increased significantly in the early stage of inflammation. Following an extended period of time, the levels decreased $(\mathrm{P}<0.001 ;$ Figs. 4 and 5).

Effects of melatonin on apoptosis in the inflammatory response. The expression of proteins associated with apoptosis was detected using western blot analysis with antibodies

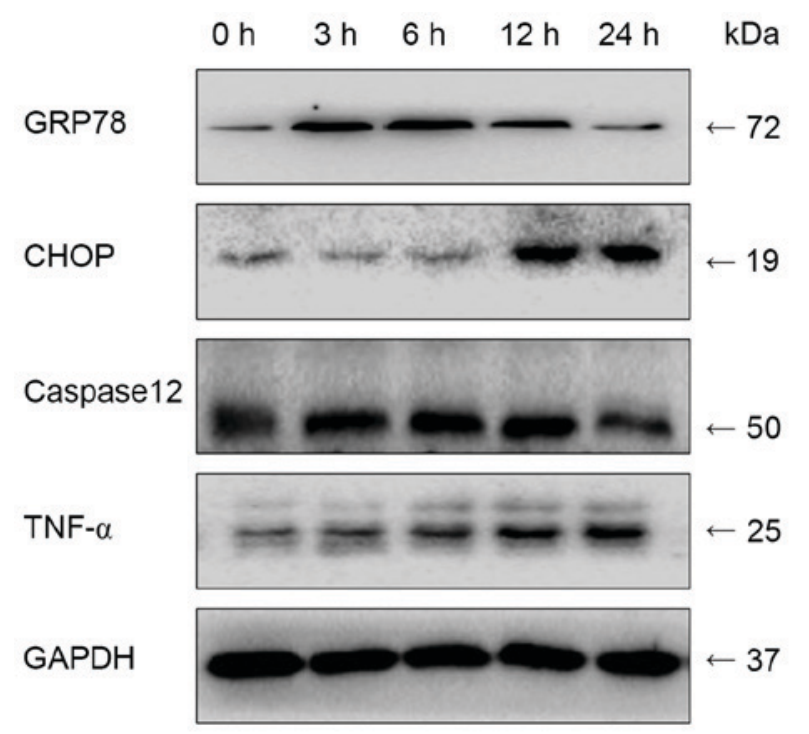

Figure 4. Effects of LPS on the ERS pathway at different durations of treatment, determined using western blot analysis. The cells were treated with $1 \mu \mathrm{g} / \mathrm{ml} \mathrm{LPS}$ for $0,3,6,12$ and $24 \mathrm{~h}$. The cultured cells were collected to extract proteins for western blot analysis. The expression levels of ERS-associated molecules were increased in the early stage of inflammation. At a certain time-point, expression levels declined. ERS, endoplasmic reticulum stress; LPS, lipopolysaccharide; GRP78, glucose-regulated protein 78; CHOP, $\mathrm{C} / \mathrm{EBP}$ homologous protein; TNF- $\alpha$, tumor necrosis factor- $\alpha$.

against Bcl-2, Bax, pro-caspase 3 and cleaved-caspase 3. As shown in Fig. 6, the levels of Bax and cleaved-caspase 3 were increased significantly in the LPS-treated group, whereas the levels of Bcl-2 and pro-caspase 3 were decreased, compared with those in the control or MLT-treated groups. Therefore, inflammation promoted apoptosis, whereas treatment with MLT reduced apoptosis, compared with treatment with LPS. 
A

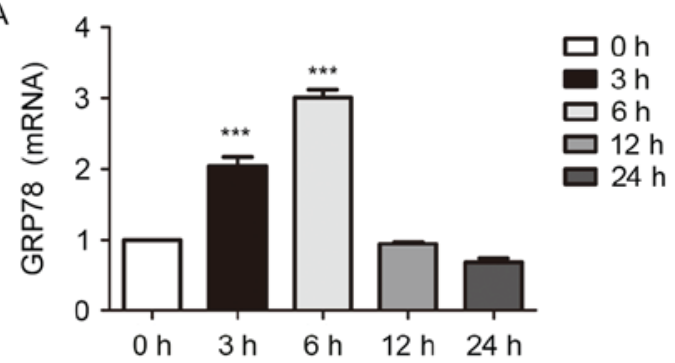

C

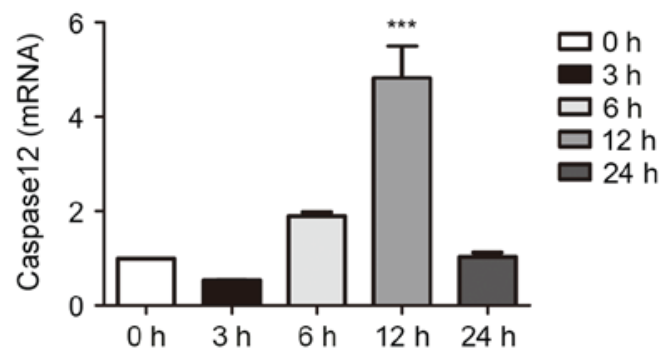

B

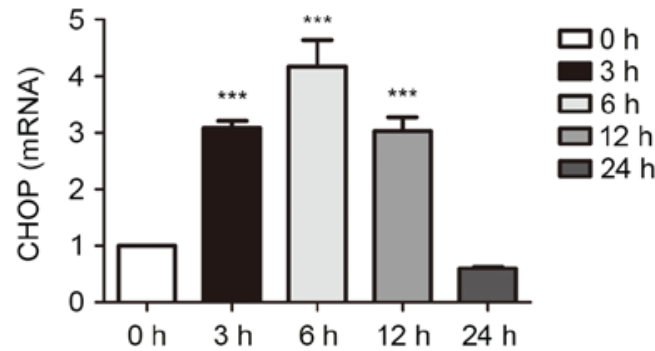

D

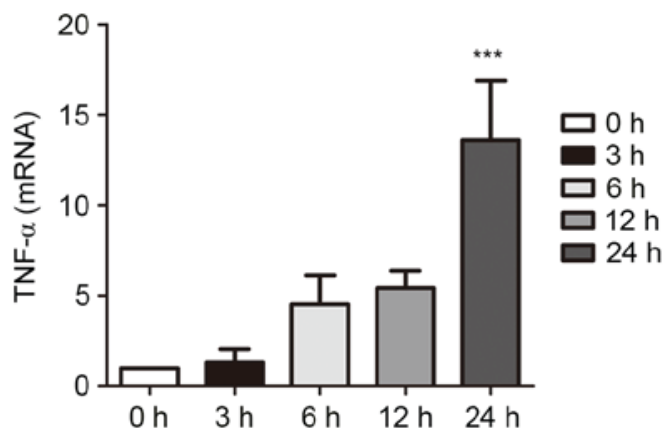

Figure 5. Effects of LPS on the endoplasmic reticulum stress pathway at different durations of treatment, determined using RT-qPCR analysis. RNA was collected for RT-qPCR analysis of (A) GRP78, (B) CHOP, (C) caspase 12 and (D) TNF- $\alpha{ }^{* * * *}$ P<0.001 vs. 0 h. LPS, lipopolysaccharide; RT-qPCR, reverse transcription-quantitative polymerase chain reaction; GRP78, glucose-regulated protein 78; CHOP, C/EBP homologous protein; TNF- $\alpha$, tumor necrosis factor- $\alpha$.

\section{Discussion}

In the present study, it was demonstrated that MLT had an anti-inflammatory effect via ERS in RAW264.7 macrophages. ERS was activated in the early stages of inflammation induced by LPS. Treatment with MLT significantly inhibited the expression of inflammatory cytokines and ERS-associated molecules, and exerted a protective effect by suppressing apoptosis of the cells.

Oztekin et al (19) found elevated levels of TNF- $\alpha$ following resection of the pineal gland and MLT inhibited the expression of TNF- $\alpha$. However, in the present study, the trend was less marked in the results of the western blot analysis, compared with that using ELISA. Therefore, it was hypothesized that MLT may have an effect on the release of TNF- $\alpha$ rather than its expression levels. This may be a focus of direction in future investigations.

Stimulation by external factors, including oxidative stress, calcium ion imbalance and lecithin synthesis disorder, can lead to numerous unfolded proteins within the ER, triggering ERS, which is an early self-protection mechanism against exogenous stress within cells. When the damage to the ER becomes irreversible and the cell cannot return to its normal function, the apoptotic signaling pathway is initiated, and the cell eventually triggers programmed cell death, or apoptosis. The apoptosis induced by ERS occurs through various events involving ERS-associated molecules, including CHOP and caspase 12, which promote apoptosis, and the expression of survival molecules, including activation of growth arrest and DNA damage protein 34 and binding immunoglobulin protein. There are several mechanisms involved in the interaction between inflammation and ERS. In pathological conditions, activated macrophages can secrete various inflammatory molecules and stimulate the activation of other cells. In mammals, however, the stress reaction, induced by the expression of stress proteins, is reversible and protects the cells from the effects of the external environment (20).

A previous study found that UPR signaling pathways can activate NF-кB. Kaneko et al (21) showed that the activation of IRE1a in the cytoplasm was associated with TNF receptor-2,

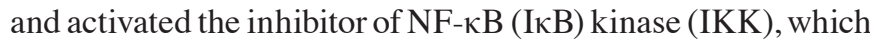
phosphorylated I $\kappa \mathrm{B}$. The phosphorylation of I $\kappa \mathrm{B}$ targeted it for ubiquitination and protease degradation. This caused the release of NF-кB, which relocated into the nucleus and initiated the transcription of associated genes. When inflammation and infection activate ERS, the PERK pathway is activated, the transcription of I $\kappa \mathrm{B}$ is reduced, and nuclear NF- $\kappa \mathrm{B}$ increases and regulates transcription (22). Similarly, ATF6 can also activate the NF- $\mathrm{BB}-\mathrm{IKK}$ signaling pathways. Unfolded proteins are transferred from the ER to the Golgi apparatus, where SP1 and SP2 divide ATF6 into ATF6a and ATF6b, resulting in the activation of these nuclear transcription factors (23). Protein kinase B (AKT) exists in the ER during ERS, and treatment with ATF6 small interfering RNA inhibits the phosphorylation of AKT, which affects the expression of downstream targets, including NF- $\kappa$ B. This shows that ATF6 can activate the inflammatory response through the AKT-NF- $\mathrm{kB}$ signaling pathway (24). There is also evidence that ER overload, rather than $\mathrm{Ca}^{2+}$ or reactive oxygen species (ROS) stress, can lead to activation of NF- $\mathrm{KB}$ in the classic UPR.

The action of heat shock proteins (HSPs) can also relieve inflammation. In cells, HSPs are important in the process of recovery to reduce irritation and damage. HSPs promote the synthesis of proteins in the ER, and their folding and translocation in the cell membrane (25). GRP78 and other GRPs are important partners of ER proteins, and they belong to the HSP protein family. GRPs, particularly GRP78, are essential in the condition of ERS (26). 
A

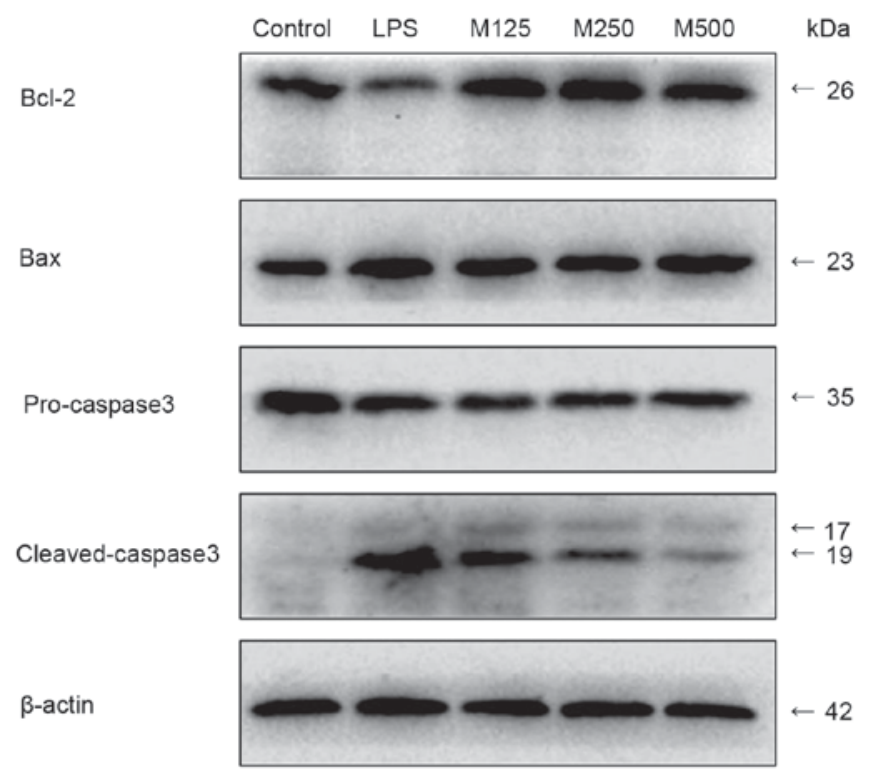

B
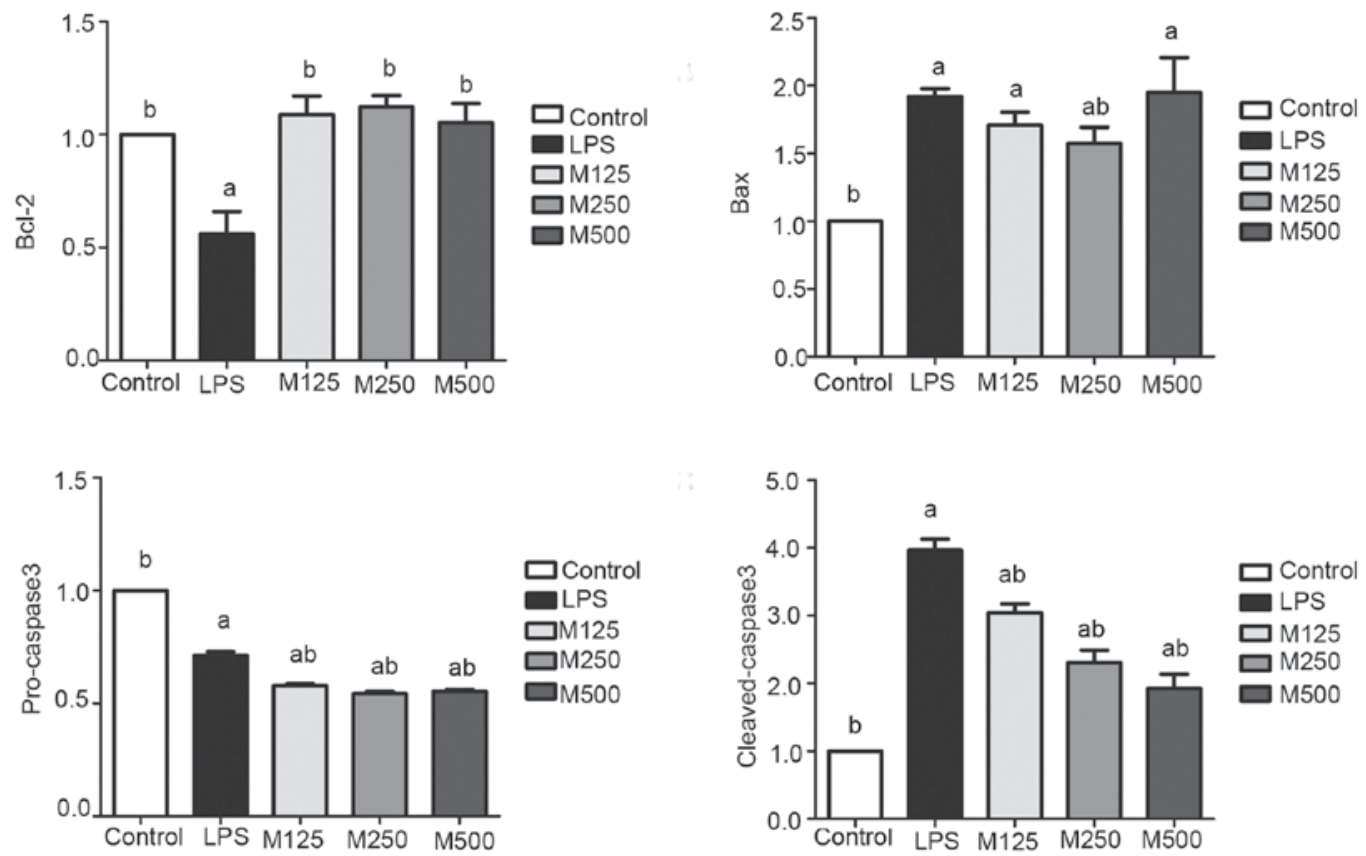

Figure 6. Effects of melatonin on apoptosis in the inflammatory response. Following treatment with or without melatonin and stimulation by LPS for $6 \mathrm{~h}$, cell proteins were extracted for (A) western blot analysis that was subsequently quantified with (B) anti-Bcl-2, anti-Bax, anti-caspase 3 and anti- $\beta$-actin antibodies ${ }^{\mathrm{a}} \mathrm{P}<0.05$ vs. control group; ${ }^{\mathrm{P}} \mathrm{P}<0.05$ vs. LPS group. LPS, lipopolysaccharide; M125, $125 \mu \mathrm{mol} / 1$ melatonin; M250, $250 \mu$ mol/1 melatonin; M500, 500 $\mu$ mol/1 melatonin; Bcl-2, B-cell lymphoma 2; Bax, Bcl-2-associated X protein.

The association between ERS and inflammation is not unilateral, and inflammatory cytokines can also activate ERS. The processing of TNF- $\alpha$ in fibrosarcoma cells from mice can cause UPR activation, as indicated by the increased expression of X-box binding protein 1, GRP78 and the phosphorylation of eukaryotic initiation factor 2 (eIF2) (27). TNF- $\alpha$, IL-1 $\beta$ and IL-6 can also lead to ERS in liver cells and cause the activation of cAMP-responsive element-binding protein $\mathrm{H}$, thus mediating the URP. This mechanism may be associated with inflammatory factors promoting calcium ion release and the accumulation of ROS in the ER, protein misfolding and mitochondrial metabolism imbalance (28). By contrast, ERS is also involved in the regulation of the inflammatory response. The activation of CHOP, induced by the PERK/eIF-2olved in, has been shown to have a negative regulatory role in inflammation (29). A study by Ho et al showed that LPS elicited inducible nitric oxide synthase (iNOS) in murine RAW264.7 cells, and tunicamycin and brefeldin A, two ER stressors, attenuated it. This indicated that multiple mechanisms are involved in the inhibition of LPS-induced gene expression of iNOS by ER stressors (30). In the present study, MLT attenuated the inflammatory response by inhibiting the activation of ERS in 
RAW264.7 macrophages. Therefore, the two conclusions are consistent and mutually supportive.

In conclusion, increasing the protein folding ability in the ER can prevent the accumulation of misfolded and toxic proteins. When the UPR cannot restore the protein folding in the ER, the cells use the three branches of the UPR to activate apoptosis. CHOP cannot directly induce apoptosis, but the degradation of anti-apoptotic proteins, including $\mathrm{Bcl}-2$, and the increased expression of apoptosis-promoting proteins, including Bax, activate the caspase cascade and lead to apoptosis. In the present study, ERS was activated in RAW264.7 macrophages under the condition of LPS-induced inflammation. At the LPS concentration of $1 \mu \mathrm{g} / \mathrm{ml}$, irreversible cell damage led to apoptosis through a reduction in the levels of Bcl-2 and increases in the levels of Bax and caspase 3. MLT decreases the expression of Toll-like receptor 3 (TLR3)-mediated inflammatory factor via inhibiting the activation of NF- $\mathrm{KB}$, and modulates TLR4-mediated inflammatory genes through the TIR-domain-containing adapter-inducing interferon $\beta$ - and MyD88-dependent signaling pathways in LPS-stimulated RAW264.7 cells $(31,32)$. However, the mechanism of action underlying MLT and ERS remains to be elucidated. In the present study, MLT reduced the inflammatory response, decreased the expression of ERS-associated proteins and inhibited cell apoptosis. Therefore, it was concluded that MLT attenuated the inflammatory response by inhibiting the activation of ERS and suppressing apoptosis of the RAW264.7 macrophages. These results provide a novel line of investigation for the anti-inflammatory effect of MLT, which uses the ERS pathway to reduce the effects of inflammation. Clinically, it is essential to intervene in the ERS pathway to enhance the therapeutic effect of anti-inflammatory drugs. However, the present study had limitations; it did not explain whether ERS was a cause or an effect of inflammation, nor did it identify the optimal concentration of MLT. Therefore, subsequent investigations aim to focus on answering the above questions.

\section{Acknowledgements}

All the authors would like to thank Mr. Weimin Li at Department of Gastroenterology (The Affiliated Hospital of Hangzhou Normal University, Hangzhou, China) for technical support.

\section{Funding}

The present study was supported by The Science and Technology Bureau of Wenzhou, Zhejiang Province, China (grant no. 2014S0192) and the Public Projects of Zhejiang Province (grant no. 2016C33215).

\section{Availability of data and materials}

The analyzed data sets generated during the study are available from the corresponding author on reasonable request.

\section{Authors' contributions}

YC performed the research and wrote this study; JW designed this study; JZ analyzed the data, revised and translated the study; QZ and YS collected information, and YJ advised on assay performance.

\section{Ethics approval and consent to participate}

Not applicable.

\section{Consent for publication}

Not applicable.

\section{Competing interests}

The authors declare that they have no competing interests.

\section{References}

1. Schröder M: Endoplasmic reticulum stress responses. Cell Mol Life Sci 65: 862-894, 2008.

2. Shen X, Zhang K and Kaufman RJ: The unfolded protein response-a stress signaling pathway of the endoplasmic reticulum. J Chem Neuroana 28: 79-92, 2004.

3. Boyce M and Yuan J: Cellular response to endoplasmic reticulum stress: A matter of life or death. Cell Death Differ 13: 363-373, 2006.

4. Rutkowski DT and Kaufman RJ: A trip to the ER: Coping with stress. Trends Cell Biol 14: 20-28, 2004.

5. Mori K: Tripartite management of unfolded proteins in the endoplasmic reticulum. Cell 101: 451-454, 2000.

6. Ron D: Translational control in the endoplasmic reticulum stress response. J Clin Invest 110: 1383-1388, 2002.

7. Hotamisligil GS: Endoplasmic reticulum stress and the inflammatory basis of metabolic disease. Cell 140: 900-917, 2010.

8. Slominski A, Tobin DJ, Zmijewski MA, Wortsman J and Paus R: Melatonin in the skin: Synthesis, metabolism and functions. Trends Endocrinol Metab 19: 17-24, 2008.

9. Hemadi M, Saki G, Shokri S and Ghasemi FM: Follicular dynamics in neonate vitrified ovarian grafts after host treatment with melatonin. Folia Morphol (Warsz) 70: 18-23, 2011.

10. do Carmo Buonfiglio D, Peliciari-Garcia RA, do Amaral FG, Peres R, Nogueira TC, Afeche SC and Cipolla-Neto J: Early-stage retinal melatonin synthesis impairment in streptozotocin-induced diabetic wistar rats. Invest Ophthalmol Vis Sci 52: 7416-7422, 2011.

11. Cohen Engler A, Hadash A, Shehadeh N and Pillar G: Breastfeeding may improve nocturnal sleep and reduce infantile colic: Potential role of breast milk melatonin. Eur J Pediatr 171: 729-732, 2012.

12. Carpentieri A, Díaz de Barboza G, Areco V, Peralta López M and Tolosa de Talamoni N: New perspectives in melatonin uses. Pharmacol Res 65: 437-444, 2012.

13. Forman K, Vara E, García C, Kireev R, Cuesta S, Acuña-Castroviejo D and Tresguerres JA: Beneficial effects of melatonin on cardiological alterations in a murine model of accelerated aging. J Pineal Res 49: 312-320, 2010.

14. Wang YT, Chen SL and Xu SY: Effect of melatonin on the expression of nuclear factor-kappa B and airway inflammation in asthmatic rats. Zhonghua Er Ke Za Zhi 42: 94-97, 2004 (In Chinese).

15. Wu JS, Wu JM, Wang D, Huang QK, Chen XR, Huang ZM, Lin XF, Chen MX and Han QX: Protective effect of melatonin on stress ulcer in rats by nuclear factor- $\kappa \mathrm{B}$. Chin J Digest 25: 107-109, 2005 (In Chinese).

16. Mei Q, Xu JM, Zhao ZH, Wu J, Hu YM and Xu XH: Effect of melatonin on expression of inflammatory cytokines in immune colitis. Chin J Microbiol Immunol 24: 711-714, 2004 (In Chinese).

17. Livak KJ and Schmittgen TD: Analysis of relative gene expression data using real-time quantitative PCR and the 2(-Delta Delta C(T)) method. Methods 25: 402-408, 2001.

18. Shao BL, Zhang YX, Wang KJ, Ying F, Sun XC and Wu JS: Melatonin reduces the production of NO and ROS in peritoneal macrophages by inhibiting p38 pathway. J Med Res 44: 63-67, 2015 (In Chinese). 
19. Oztekin E, Mogulkoc R,Baltaci AK and Tiftik AM: The influence of estradiol and progesterone and melatonin supplementation on TNF-alpha levels in ovariectomized and pinealectomized rats. Acta Biol Hung 57: 275-281, 2006.

20. Hayakawa K, Hiramatsu N, Okamura M, Yamazaki H, Nakajima S, Yao J, Paton AW, Paton JC and Kitamura M: Acquisition of anergy to proinflammatory cytokines in nonimmune cells through endoplasmic reticulum stress response: A mechanism for subsidence of inflammation. J Immunol 182: 1182-1191, 2009

21. Kaneko M, Niinuma $Y$ and Nomura $Y$ : Activation signal of nuclear factor-kappa B in response to endoplasmic reticulum stress is transduced via IRE1 and tumor necrosis factor receptor-associated factor 2. Biol Pharm Bull 26: 931-935, 2003.

22. Deng J, Lu PD, Zhang Y, Scheuner D, Kaufman RJ, Sonenberg N Harding HP and Ron D: Translational repression mediates activation of nuclear factor kappa B by phosphorylated translation initiation factor 2. Mol Cell Biol 24: 10161-10168, 2004.

23. Haze K, Yoshida H, Yanagi H, Yura T and Mori K: Mammalian transcription factor ATF6 is synthesized as a transmembrane protein and activated by proteolysis in response to endoplasmic reticulum stress. Mol Biol Cell 10: 3787-3799, 1999.

24. Nakajima S, Hiramatsu N, Hayakawa K, Saito Y, Kato H, Huang T, Yao J, Paton AW, Paton JC and Kitamura M: Selective abrogation of BiP/GRP78 blunts activation of NF- $\kappa \mathrm{B}$ through the ATF6 branch of the UPR: Involvement of C/EBP $\beta$ and mTOR-dependent dephosphorylation of Akt. Mol Cell Biol 31: 1710-1718, 2011.

25. Hartl FU and Hayer-Hartl M: Molecular chaperones in the cytosol: From nascent chain to folded protein. Science 295: 1852-1858, 2002.
26. Lee AS: The glucose-regulated proteins: Stress induction and clinical applications. Trends Biochem Sci 26: 504-510, 2001.

27. Zhang K, Shen X, Wu J, Sakaki K, Saunders T, Rutkowski DT, Back SH and Kaufman RJ: Endoplasmic reticulum stress activates cleavage of CREBH to induce a systemic inflammatory response. Cell 124: 587-599, 2006.

28. Lin W, Harding HP, Ron D and Popko B: Endoplasmic reticulum stress modulates the response of myelinating oligodendrocytes to the immune cytokine interferon-gamma. J Cell Biol 169: 603-612, 2005.

29. Garg AD, Kaczmarek A, Krysko O, Vandenabeele P, Krysko DV and Agostinis P: ER stress-induced inflammation: Does it aid or impede disease progression? Trends Mol Med 18: 589-598, 2012

30. Ho HJ, Huang DY, Ho FM, Lee LT and Lin WW: Inhibition of lipopolysaccharide-induced inducible nitric oxide synthase expression by endoplasmic reticulum stress. Cell Signal 24: 2166-2178, 2012.

31. Huang SH, Cao XJ and Wei W: Melatonin decreases TLR3-mediated inflammatory factor expression via inhibition of NF-kappa B activation in respiratory syncytial virus-infected RAW264.7 macrophages. J Pineal Res 45: 93-100, 2008.

32. Xia MZ, Liang YL, Wang H, Chen X, Huang YY, Zhang ZH, Chen YH, Zhang C, Zhao M, Xu DX and Song LH: Melatonin modulates TLR4-mediated inflammatory genes through MyD88- and TRIF-dependent signaling pathways in lipopolysaccharide-stimulated RAW264.7 cells. J Pineal Res 53: 325-334, 2012. 